\title{
Calcific uremic arteriolopathy
}

\section{Olanrewaju A. Olaoye and Abhilash Koratala*}

Division of Nephrology, Hypertension and Renal Transplantation, University of Florida, Gainesville, FL, USA

*Correspondence address. Division of Nephrology, Hypertension and Renal Transplantation, University of Florida, 1600 SW Archer rd, Room CG 98, Communicore building, Gainesville, FL 32610, USA. Tel: +1-352-294-8694; Fax: +1-352-392-3581; E-mail: akoratsla@ufl.edu

\begin{abstract}
Calcific uremic arteriolopathy (CUA) or Calciphylaxis is a rare disease typically seen in end stage renal disease patients on dialysis and is associated with high mortality rates, mainly because of sepsis. Medial calcification of the dermal arterioles is the characteristic histologic finding together with vascular thrombosis and ischemic necrosis. CUA involves legs, abdomen and gluteal region in majority of the patients. Herein, we present a case of CUA of the glans penis in a uremic patient. Unfortunately, the patient died of sepsis in a few weeks after initiating dialysis.
\end{abstract}

An 81-year old white man with history of hypertension, hyperlipidemia and chronic kidney disease (CKD) Stage 5 was admitted to the hospital for uremic symptoms necessitating initiation of haemodialysis. He was recommended to start dialysis $\sim 8$ months ago but refused at that time and was lost to follow-up. The patient complained of pain and redness of the glans penis that first appeared 3 weeks ago. At that time, he was treated for possible balanitis with antibiotics and had partial relief. He

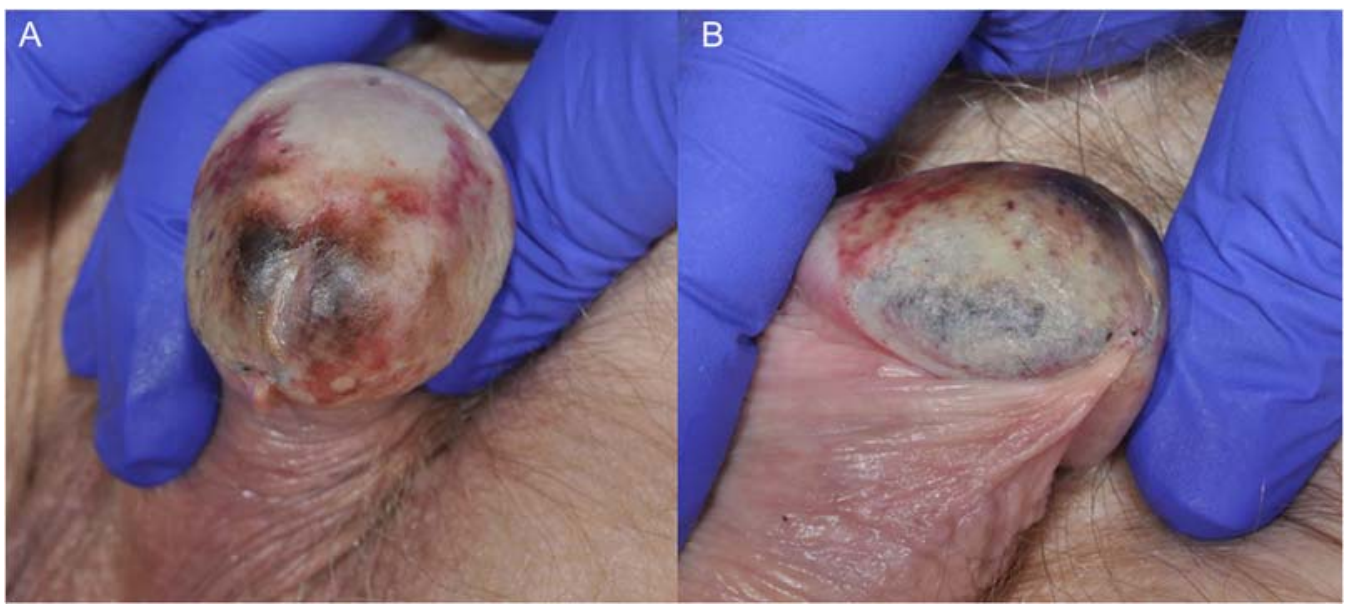

Figure 1: (A and B) Purpuric patches, erosions and superficial necrosis of penile glans suggestive of CUA.

This is an Open Access article distributed under the terms of the Creative Commons Attribution Non-Commercial License (http://creativecommons.org/ licenses/by-nc/4.0/), which permits non-commercial re-use, distribution, and reproduction in any medium, provided the original work is properly cited. For commercial re-use, please contact journals.permissions@oup.com 


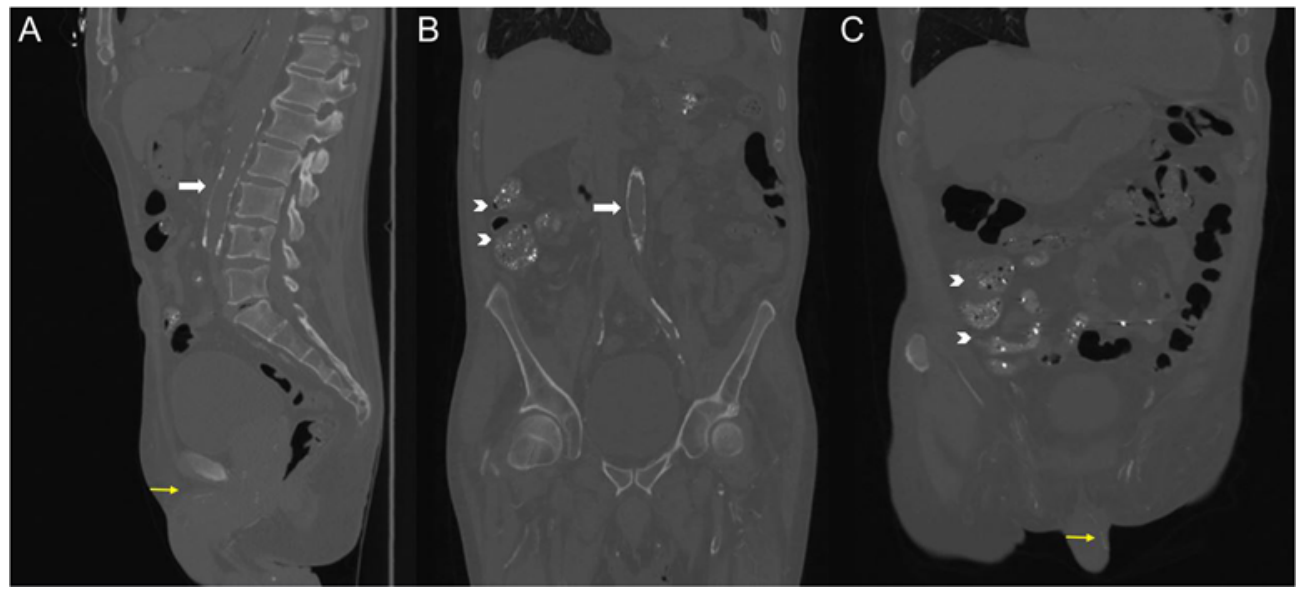

Figure 2: Sagittal (A), coronal (B) and oblique (C) views of non-contrast CT scan of the abdomen demonstrating extensive calcification of the aorta (white arrows) and pudendal vessels (yellow arrows). The intraluminal densities in the bowel that were incidentally found (indicated by chevrons) are likely due to lanthanum carbonate, which is a metal-based phosphate binder.

denied having any fever, chills, difficulty urinating or discharge from the urethra. On examination, he had purpuric patches, erosions and superficial necrosis of penile glans with minimal tenderness [Fig. 1]. There were no similar lesions elsewhere in the body. His labs at presentation were significant for serum creatinine of $12.7 \mathrm{mg} / \mathrm{dl}(0.8-1.2)$ with an estimated glomerular filtration rate of $4.1 \mathrm{ml} / \mathrm{min} / 1.73 \mathrm{~m}^{2}$ using MDRD equation, potassium $6.8 \mathrm{mmol} / 1$ (3.4-5.1), phosphate $8.4 \mathrm{mg} / \mathrm{dl}$ (2.7-4.5), calcium $9.8 \mathrm{mg} / \mathrm{dl}$ (8.4-10.2), albumin $2.6 \mathrm{~g} / \mathrm{dl}$ (3.5-5) and parathyroid hormone $1131 \mathrm{pg} / \mathrm{ml}$ (150-300 for end stage renal disease (ESRD)). He was not taking his phosphate binder and vitamin D analogue as prescribed. CT scan of the abdomen showed extensive calcification of the aorta and pudendal vessels [Fig. 2]. Based on the clinical features and imaging evidence of vascular calcification, we diagnosed him with calcific uremic arteriolopathy (CUA) of the penis. Though the definitive diagnosis of CUA requires skin biopsy, it is not recommended for penile lesions because of the risk for progression of necrosis [1].

CUA or Calciphylaxis is a rare and potentially fatal condition that presents with skin ischemia and necrosis, typically seen in ESRD patients on dialysis. Histologically, it is characterized by medial calcification of dermal arterioles. CUA commonly involves legs, abdomen and gluteal region, and rarely locations such as breast [2] and penis. Risk factors include White race, CKD-mineral bone disease axis abnormalities (hyperparathyroidism, hypercalcemia and hyperphosphatemia), obesity, hypercoagulable conditions including protein $\mathrm{C}$ and $\mathrm{S}$ deficiency, diabetes mellitus, hypoalbuminemia, warfarin use and longer dialysis vintage of over 6-7 years [3]. Our patient had several of these risk factors including white race, hyperphosphatemia, severe hyperparathyroidism, hypoalbuminemia and a uremic milieu. Protein C\&S deficiency and antiphospholipid syndrome were excluded by appropriate lab tests. He was managed with intensification of dialysis regimen, phosphate lowering therapy, cinacalcet (calcimimetic agent) and intravenous sodium thiosulfate therapy. However, the patient died of sepsis $\sim 6$ weeks after starting dialysis.

The important take-home points from our case include (i) CUA is a rare and highly morbid metabolic complication of kidney disease and (ii) Glans penis is a rare site of involvement and the diagnosis is usually made clinically without biopsy.

\section{ACKNOWLEDGEMENTS}

None.

\section{CONFLICT OF INTEREST STATEMENT}

None declared.

\section{FUNDING}

None.

\section{ETHICAL APPROVAL}

None required.

\section{CONSENT}

Informed consent obtained from the patient for publication.

\section{GUARANTOR}

Abhilash Koratala, M.D.

\section{REFERENCES}

1. Cimmino CB, Costabile RA. Biopsy is contraindicated in the management of penile calciphylaxis. J Sex Med 2014;11: 2611-7.

2. Koratala A, Ejaz AA, Mohandas R. Calciphylaxis of the breast: a rare metabolic complication of kidney disease. BMJ Case Rep 2016. doi:10.1136/bcr-2016-216665.

3. Nigwekar SU, Kroshinsky D, Nazarian RM, Goverman J, Malhotra R, Jackson VA, et al. Calciphylaxis: risk factors, diagnosis, and treatment. Am J Kidney Dis 2015;66:133-46. 